\title{
Augmented Reality Technology and Its Game Application Research
}

\author{
Duan Ganghai $^{1, \text { a, }}$, Han Minglei ${ }^{1, b}$, Zhao Weiqi ${ }^{1, ~ b}$, Dong Tianhao ${ }^{1, b}$ and Xu Tingting ${ }^{1, b}$ \\ ${ }^{1}$ College of Computer Science and Technology, Jilin University, Changchun, China \\ adgh_sea@163.com, ${ }^{\mathrm{b}}$ css4142@163.com \\ *corresponding author
}

Keywords: augmented reality, computer vision, AR games

\begin{abstract}
Augmented reality technology is one of the hottest topics in the field of computer vision. This paper systematically reviews the research status of vision-based augmented reality technology. First, it outlines the current research status and application of augmented reality technology; secondly, it elaborates the key technologies of augmented reality, including display technology, tracking registration technology, interaction technology, and calibration technology. Finally, according to the different working methods, All AR games are divided into two categories, basing on fixed patterns and basing on geographical location, giving a brief introduction to the AR games that stand out on the market.
\end{abstract}

\section{Introduction}

Augmented Reality (AR), also known as mixed reality technology, combines virtual objects created by computers with the real world to construct a virtual space with virtual reality, which is a new development based on virtual reality. Technology is also one of the most high-tech concerns today. Milgram defines a continuum from the real to the virtual environment ${ }^{[1]}$.

As augmented reality technology has huge application value on the mobile phone platform, mobile internet terminal manufacturers, chip manufacturers and various industry giants attach great importance to this technology and actively promote its development. Especially in the digital game industry, the use of augmented reality technology is one of the hot spots. Many international game companies have started the design of AR games.

\section{Research Status}

As early as the 1990s, the exploration of augmented reality technology has been started, but this technology has really developed since the last few years, mainly because of the role of mobile terminals, especially the rapidly development of current smart-phones. Therefore, in recent years, this game has become increasingly popular. The important content that needs to be focused on is how to make the real game better and make the future gameplay more unique and interesting.

There are already many practical AR applications in the world. In Germany, engineers and technicians, when installing, repairing, and adjusting the machine, use the head-mounted display to completely present the internal structure of the machine, as well as its related information and data. Sweden's IKEA "Home Guide" allows users to use IKEA's official magazines as identification cards to display IKEA products in the magazine and place these products through augmented reality technology in every corner of the home. With the Tokyo Aquarium App in Tokyo, users can open the app at anywhere in Tokyo, and the cute aquarium penguin will navigate and guide users to the aquarium. There are also interesting AR apps like Sophie Rabbit, iButterfly, McDonald's GOL!, Toyota 86 and more.

AR technology has a very good prospect in current world . In a research report, the Bartel Institute of the United States listed 10 technological trends with the most strategic significance in 2020. These trends will determine the characteristics of social development in the coming period, No. 10 is reality technology. Google executives, including CEOs Sundar Pichai and Clay Bavor, 
have privately stated that AR or "hybrid" reality, where digital information and images overlap or alternate with people's vision of the real world, will be a bigger market than VR in the long run.

\section{Key Technologies for Augmented Reality}

Augmented reality technology is developed on the basis of computer graphics, computer graphics processing, and machine learning ${ }^{[2]}$. It superimposes the original virtual objects onto the real world through some computer technologies, so human beings will get a perfect realistic sensory experience. The construction of an AR system needs to solve many key technical problems, which mainly include display technology, tracking registration technology, interaction technology and calibration technology.

\subsection{Display methods}

Vision is the most important way for human beings to obtain information from the external environment. Therefore, display technology is one of the key technologies in augmented reality technology. The purpose of display technology is using computer-generated virtual images into real-life environments. Display technologies for enhanced display systems include head-mounted displays, hand-held displays, projection displays, etc. ${ }^{[3]}$.

\subsubsection{Head-Mounted Display}

This monitor is worn on the head and displays the image in front of the eyes. It is mainly divided into two kinds, video perspective headset display based on camera principle and optical perspective headset display based on optical principle.

The video see-through head-mounted display acquires real-time images through the camera on the device, and the three-dimensional objects generated by the image processing module and the virtual rendering module are merged with each other and finally displayed on the head-mounted display.

The optical see-through head-mounted display utilizes the principle of light reflection. A translucent optical combiner is placed in front of the user's eyes. The user can see the virtual threedimensional object and the display object merge into each other. This image has not been processed by the image and it is the user's eyes that see the real scene ${ }^{[4]}$.

\subsubsection{Hand-Held Display}

This is a flat LCD display that uses bundled cameras to provide video perspective based enhancements, and the hand-held display acts as a window or magnifying glass to display real objects covered with AR. Smart-phones generally have a camera, built-in GPS, and built-in inertial sensors, magnetic sensors, etc. At the same time, they have higher resolution displays, small size and easy to carry. They are ideal devices for augmented reality technology development.

\section{1.3 Projection Display}

The required virtual information is directly projected onto the real object for enhancement. In the simplest case, the enhanced information is directly projected onto the surface of the object so that no special glasses are needed ${ }^{[5]}$. The other is a head-mounted projector that projects an image onto an object in the real world in the direction of the viewer's line of sight. The target object is coated with a retro-reflective substance that reflects the light back along the incident angle. By using the respective HMD system, multiple users can simultaneously see different images on the same projection target ${ }^{[6]}$. 


\subsection{Track Registration Technology}

In order to achieve the ultimate goal of realizing the exact integration of real scenes and virtual objects, the augmented reality system needs to establish the conversion relationship between the virtual space coordinate system and the real space coordinate system so that the virtual object can be merged with the correct position of the real world. This process is Registration. Since the observer's position will constantly change, the system will reconstruct the coordinate system relationship in real time according to the observer's perspective. This process is Tracking ${ }^{[7]}$.

There are mainly three tracking and positioning technologies in augmented reality systems. Tracker-based registration, using tracking sensors; visual tracking registration, using computer vision systems combined with specific algorithms; composite registration method, tracker registration and visual registration combined.

\subsubsection{Tracker Registration Method}

Hardware tracker is a technique for tracking location. According to the different signal transmission devices, it can be divided into seven types: optical systems, GPS, ultrasonic, inertial devices, gyroscopes, magnetic fields, and mechanical devices. However, these methods have certain limitations, the accuracy and range can not meet the requirements, and they are also vulnerable to external interference.

\subsubsection{Visual Tracking Registration}

Based on video detection, tracking and positioning technology is also the most used tracking method in augmented reality systems, which can be further divided into two different video detection technologies based on markers and natural features. The former needs to set markers in the scene in advance, and the latter needs to detect natural features in the real scene.

\subsubsection{Compound Registration}

Hybrid tracking and positioning technology. This is also the method most current augmented reality systems use. First, it uses the electronic compass and GPS for initial positioning, and then uses the video detection method to achieve more accurate tracking and positioning. Therefore, it is more accurate than the previous two tracking registration methods.

\subsection{Interaction Technology}

Interaction technology refers to the technology that enables people to interact with computers through effective computer input and output devices. It includes a large number of relevant information, prompts, and instructions provided by the machine through the output or display device, as well as information input by the person through the input device to the machine to answer questions, hints, and instructions. There are mainly three kinds of interaction modes used in augmented reality technology: spatial point interaction mode, command interaction mode, and interaction mode using special tools ${ }^{[8]}$. In an augmented reality scenario, if a user issues an instruction to a virtual scene object, the virtual item can provide feedback to the user so that the advantages of the enhanced scene can be fully utilized.

\subsection{Calibration Technology}

The calibration technique is to realize the mapping from the image plane pixel point to the real world space coordinate point, so as to obtain the information that the virtual object accurately registers to the scene. Calibration technology is the link between the virtual world and the real world. Its quality directly determines the stability of virtual reality registration and the authenticity 
of virtual reality integration in the future ${ }^{[9]}$. In order to generate accurate positioning, the AR system needs a lot of calibration. The measured values include camera parameters, sight zone, sensor offset, object positioning and deformation, etc. Currently, AR calibration uses the camera calibration principle, and many manual AR calibration technologies. One way to avoid calibration is to develop calibration freedom Drawer.

\section{Augmented Reality Based Game Classification}

The method of reluctant reality is based on the "camera acquires images - acquires special marks in images - calculates 3D information and overlays virtual objects". According to the different types of marks, the current AR applications can be divided into two kinds: Basing on fixed patterns and basing on geographic location .

Based on the fixed pattern, computer realizes the recognition of the fixed pattern by the camera, and application will be triggered in real time after the recognition is successful. Based on geographical location, GPS positioning is used to place identification points at fixed locations. Only the positioning technology and image processing technology can be used in real world.

According to the role of bare reality technology in the application, augmented reality games are divided into augmented reality content type, augmented reality control type, and hybrid type. Augmented reality content type means that the actual content in the game uses augmented reality technology; augmented reality control type means that players must use augmented reality technology to achieve game operations; in hybrid type, augmented reality technology will be applied to Content scenes and operations. For augmented reality games, there are huge differences in the content and presentation of different games, so this classification is not accurate. Because the AR games of image processing are often very different in requirements, the selected positioning technology is also very different. A brief introduction will be made to the AR games that are prominent in the market for image processing.

\subsection{Magicplan}

Magicplan is a software that uses the camera of the mobile phone to make the floor plan of the room, can measure the room and draw the floor plan of the room. Use the camera to mark the corners of the room one by one. At the same time, the software supports marking the position of the door, so that the area will not be wrong. Looking around, the software can draw all the rooms into place. The figure drawn is professional enough, including the length and width of each location.

\subsection{Paint Space}

Paint Space is a mobile AR creative application that allows users to draw in the air, hang pictures and videos, and share their works with their friends. The Paint Screen uses a touch screen that allows every user to draw in the habit that he had developed since childhood. As people become more familiar with the possibilities that can be brought about by moving their own mobile phones, the application can help people create dynamic lines in three-dimensional space without having to bother "re-learning" new painting methods. Essentially, the Paint Space is a very futuristic experience of using a finger to paint. Many sculptors use their hands as tools - in Paint Space, the user's finger is the tool pen. The use of multitouch makes Paint Space a 1: 1 experience that users can use and touch, easily turning their fingers into powerful drawing tools.

\subsection{Jigspace}

JigSpace can be seen as a "Teach you how" training manual. Its goal is to cover all products and equipment on the planet with the help of Apple ARKit. For example, you just bought a new chair and you want some help outside the manual to build it. Then JigSpace will show the 3D model of the unassembled chair, then provide a real-time step-by-step guide so that users can observe the 
virtual structure from any angle, and finally users can copy the operation in real life. JigSpace presents the interesting science model. The application has a variety of built-in AR object models, from the human physiological structure, ancient weapons, to geological geography. When the AR model hovering over the desktop, click the arrow in the lower right corner of the screen, the user can view the human brain, alarm clock and other structures, the text within the application will provide a more detailed description, there will be an explosion map to show its internal structure. In operation, it is also convenient to observe from different perspectives. The user can use two fingers to zoom and single-finger operation to rotate.

\section{Summary}

Augmented reality technology is a new technology developed on the basis of virtual reality technology. It has been widely used in industries such as industry, agriculture and national defense. This paper summarizes the research on AR technology, including the current research status of AR technology, some key technologies for realizing AR, and summarizes the application of AR games. This lays a good foundation for the gradual application of augmented reality technology to people's daily life and entertainment. It can be expected that the seamless convergence between online and offline will usher in a new era of mobile Internet for humanity.

\section{References}

[1] Milgram P and Kishino F. A taxonomy of mixed reality visual displays[J]. IEICE Trans. Information Systems,1994, E77-D(12): 1321-1329.

[2] TangLin.Multi sensor fault detection and diagnosis mechanism based on Markoff prediction [J].Sensors and Microsystems. 2014.11(33):52-55.

[3] HouYing,Xu WeiWei.An overview of Augmented Reality Technology.[J].Computer measurement and control.2017,25(02):1-7+22.

[4] Qi Yue,Ma Hongmei. The features, key technologies and applications of augmented reality [J].Minicomputer system. 2004(05):900-903.

[5] Raskar Retal. Table-top spatially-augmented realty: bringing physical models to life with projected imagery[C].Proc.2nd Int'l Workshop Augmented Reality, 1999: 64-71.

[6] Hua Hetal. Anultra-light and compact design and implementation of head-mounted projective displays[C].Proc.IEEE Virtual Reality 2001, 2001: 175-182.

[7] Wan Yi.Application of augmented reality in smart phone interaction design[D].Master's degree thesis of North China University of Technology,2014.

[8] Sui Sai.Research on mobile augmented reality system based on Android[D].Master's degree thesis of the University of national defense science and technology,2014.

[9] Cai Pan.Research and implementation of real time interaction technology based on Augmented Reality[D].Master's degree thesis of the University of Electronic Science and technology,2012 